\title{
Longitudinal Analysis of Short-term Changes in Relationship Conflict During COVID- 19: A Risk and Resilience Perspective
}

\author{
Shawna J. Lee, ' iD Kaitlin P. Ward,' and \\ Christina M. Rodriguez ${ }^{2}$ iD
}

\begin{abstract}
This study uses a risk and resilience framework to examine short-term self-reported changes in relationship conflict early in the COVID-19 pandemic (March and April 2020). Longitudinal data from U.S. adults in a romantic relationship $(N=291)$ were collected via three waves of an online survey. Participants self-reported anxiety, depression, increased alcohol use, and dyadic coping since the pandemic. Relationship conflict variables included whether the participant reported that they and their partner "had disagreements related to the Coronavirus," "had more disagreements than usual," "had more verbal fights than usual," and "had more physical fights than usual" in the past two weeks. Analyses controlled for sociodemographic characteristics as well as days spent in lockdown and employment change due to COVID-19. Results indicated that couples' disagreement and verbal fighting scores increased from Time I to Time 2, but disagreements related to COVID-19 and physical fighting did not. Couples with higher levels of dyadic coping reported fewer fights and disagreements on average. However,
\end{abstract}

'University of Michigan, Ann Arbor, MI, USA

2University of Alabama at Birmingham, AL, USA

\section{Corresponding Author:}

Shawna J. Lee, School of Social Work, University of Michigan, 1080 South University Avenue, Ann Arbor, MI 48109-I 106, USA.

Email: shawnal@umich.edu 
dyadic coping did not buffer participants from increases in relationship conflict. Increased alcohol use since the pandemic was positively associated with disagreements related to COVID-19, disagreement scores, and verbal fighting scores. More days spent in lockdown was associated with increases in disagreements related to COVID-19. The conditions created by COVID-19 may contribute to worsening relationship conflict, even among couples who start with high levels of dyadic coping. Depression and alcohol use may contribute to poorer relationship quality during the pandemic. There is need for enhanced intervention and mental health supports to mitigate the potential effects of the pandemic on couples' relationship functioning.

\section{Keywords}

mental health, alcohol and drugs, predicting domestic violence, domestic violence

For nearly all Americans, the COVID-19 pandemic created significant disruptions to daily life. On March 16, 2020, the U.S. White House issued social distancing guidelines which instructed all Americans to stay home if they felt sick, work or engage in school from home, and avoid social gatherings of more than 10 people (The White House, Office of the Press Secretary, 2020). Quarantine and stay-at-home orders may exacerbate relationship conflict and intimate partner violence (IPV) because they result in increased exposure to an abusive partner, while also removing sources of social support during a time of adversity (Mazza et al., 2020).

Crisis periods can cultivate the conditions that exacerbate relationship conflict and IPV. Research suggests that family violence increased after previous natural disasters, such as Hurricane Katrina (Harville et al., 2011; Hawkins, 2009) and Hurricane Harvey (Serrata \& Alvarado, 2019). During the Great Recession, the incidence of IPV was twice as likely for women experiencing economic hardship compared to women who were not experiencing economic hardship (Schneider et al., 2016). The COVID-19 pandemic has been linked to worsening of other forms of family violence such as child maltreatment (Lee, Ward, Lee et al., 2021; Rodriguez et al., 2020), elder abuse (Makaroun et al., 2020), and sibling violence (Perkins et al., 2021). Early cross-sectional research during the pandemic suggested that $17 \%$ of victims of IPV reported that victimization worsened since the pandemic began, $54 \%$ conveyed no change, and, notably, $30 \%$ who were surveyed indicated victimization had lessened (Jetelina et al., 2020). Another study found 
that the stay-at-home orders in Illinois resulted in a 7.5\% increase in domestic violence-related calls for police service (Bullinger et al., 2020). While calls related to domestic violence increased, domestic violence crimes were underreported due to stay-at-home orders in March and April 2020 (Bullinger et al., 2020). Even though emergency department (ED) visits declined overall during the pandemic, one study indicated that IPV-related physical abuse and severity of injuries among IPV-related ED visits increased (Gosangi et al., 2020).

\section{Relationship Conflict During COVID-19: A Risk and Resilience Framework}

We utilized a risk and resilience framework to examine factors associated with self-reported relationship conflict during the first two months of the COVID-19 pandemic. In the context of challenging life situations, resilience has been defined as "a stable trajectory of mental health despite exposure to a serious stress" (Chen \& Bonanno, 2020, p. S51). A risk and resilience perspective highlights the confluence of risk-enhancing (e.g., unemployment, mental health problems) and protective or buffering factors (e.g., dyadic coping) that relate to functioning during a time of adversity (Chen \& Bonanno, 2020). Even though the experience of adversity related to the pandemic was widespread, with millions of Americans experiencing personal strain and economic stress, sources of resilience such as coping skills and positive relationships may contribute to resilient functioning (Chen \& Bonanno, 2020).

\section{Dyadic Coping: A Source of Resilience}

In the context of personal relationships, dyadic coping is thought to buffer individuals from relationship conflict during stressful times. Dyadic coping emphasizes that relationship functioning is enhanced when active stress management processes are enacted by both partners to restore equilibrium within the couple (Bodenmann, 1997). Dyadic coping can be differentiated from general coping because dyadic coping refers to the interplay of coping strategies between partners within a couple, often in response to external stressors (Falconier \& Kuhn, 2019; Levesque et al., 2014). Dyadic coping is an active process that engages both partners, wherein one partner responds to the stress cues of their partner in order to maintain individual and couple well-being (Bodenmann, 1997). In the present study, we examined one partner's perception of dyadic coping with their partner early during the stay-at-home orders.

Research has supported the importance of dyadic coping and its benefits to the individual and the couple during stressful times (Falconier \& Kuhn, 
2019). Self-reported dyadic coping is associated with higher levels of relationship satisfaction (Bodenmann, 2000; Levesque et al., 2014; Merz et al., 2014). More distressed couples report lower levels of dyadic coping (Bodenmann, 2000; Merz et al., 2014). Dyadic coping shows longitudinal associations with outcomes such as lower levels of divorce and separation among married couples (Bodenmann \& Cina, 2006). Dyadic coping is associated with decreased verbal aggression during times of stress (Bodenmann et al., 2010).

\section{Factors Related to IPV and Relationship Conflict}

Prior to the COVID-19 pandemic, the National Intimate Partner and Sexual Violence Survey (NISVS) estimated that approximately one in four women and one in ten men in the United States have experienced lifetime incidence of physical, emotional, sexual, or psychological intimate partner violence (Smith et al., 2018). The NISVS estimates that annually approximately 6.6\% of U.S. women experienced sexual violence, physical violence and/or stalking; and 14\% experienced psychological aggression (Smith et al., 2017). Rates for men were similar. Annually, $6.4 \%$ of U.S. men experienced sexual violence, physical violence and/or stalking, and $18 \%$ experienced psychological aggression (Smith et al., 2017).

Overall, research has suggested that economic strain is associated with elevated risk for IPV and relationship conflict (Conger et al., 2010). Poorer economic circumstances, either via chronic poverty or episodic economic strain experienced due to sudden unemployment (Stith et al., 2004), are thought to increase risk for IPV, couple conflict (Neppl et al., 2016), and poorer relationship functioning (Conger et al., 2010). Unemployment has been linked to male-perpetrated partner violence (Cunradi et al., 2009). The unemployment rate quadrupled during the U.S. stay-at-home orders (U.S. Bureau of Labor Statistics, 2020), which may contribute to heightened relationship conflict (Conger et al., 2010; Stith et al., 2004).

Mental health is another consideration. Depression (Forgey \& Badger, 2010; Spencer et al., 2019), anxiety (Spencer et al., 2019), and substance abuse (Foran \& O'Leary, 2008) are linked to IPV perpetration and victimization. Indeed, reports suggest a dramatic increase in depression among U.S. adults during COVID-19 (Lee, Ward, Chang et al.,2021; Lee, Ward, Lee et al.,2021; Twenge \& Joiner, 2021). Alcohol use is also acknowledged as a risk factor for IPV (Charles et al., 2011; Stith et al., 2004), wherein couples with greater reciprocal conflict report more problematic alcohol use (Cafferky et al., 2018; Capaldi et al., 2012). Greater alcohol consumption appears to evoke 
more severe incidents of IPV relative to other IPV episodes (Graham et al., 2011). Increased levels of alcohol use (Colbert et al., 2020) could translate into greater couple conflict.

Finally, the nature of quarantine is a unique factor that may contribute to risk for conflict (Bullinger et al., 2020; Mazza et al., 2020). Quarantine and stay-at-home orders required partners to live together in close quarters for an extended period of time, largely without direct physical contact with others. In quarantine, abuse victims lack access to extrafamilial support, which increases abusers' access to their victims (Mazza et al., 2020).

\section{The Current Study}

We focused on short-term changes in relationship conflict using survey data collected at three time points during March and April 2020. We examined (a) whether levels of relationship conflict changed across the early weeks of the pandemic and (b) whether risk and resilience factors - including dyadic coping, employment changes, mental health problems, increased alcohol use since the pandemic, and days in lockdown - were related to changes in relationship conflict (disagreements, verbal fights, and physical fights) during the first two months of the pandemic. We hypothesized that stronger dyadic coping would serve as a resilience factor and would be associated with lower levels of initial relationship conflict as well as predict individuals' lower subsequent conflict. We hypothesized that employment changes, increased alcohol use, and depression and anxiety would be associated with higher levels of relationship conflict initially and would contribute to increases in relationship conflict over time.

\section{Methods}

\section{Procedures}

Data were collected via online surveys that were administered through Prolific, a survey research company (Palan \& Schitter, 2018). The first wave of the survey (Time 1) was launched on March 24, 2020, nearly two weeks after the WHO declared that the Coronavirus was a pandemic on March 11, 2020 (WHO, 2020) and one week after the U.S. White House issued social distancing guidelines to slow the spread of COVID-19 (The White House, Office of the Press Secretary, 2020). The second wave of the survey (Time 2) was launched on April 14, 2020, and the third wave of the survey (Time 3) was launched on April 30, 2020. 
At Time 1, for initial recruitment into the study, participants were provided with a brief description of the survey via the Prolific website. In order to be eligible, individuals had to have U.S. nationality and be age 18 years or older. Participants who met the study criteria were sent an email from Prolific regarding their eligibility to participate in the survey. If they chose to participate in the survey, they were linked through the Prolific platform to a Qualtrics survey designed and managed by the research team. In Prolific, the research team set a predetermined target enrollment number. When that number was reached the survey automatically closed. At Time 1, it took less than 24 hours for the survey to reach the target enrollment number. The research team does not have information on individuals who might have viewed the study details on the Prolific platform but decided not to participate, or on individuals who wanted to participate after enrollment for the survey was already closed.

After potential participants reviewed the study information and provided informed consent, they completed the online survey. At Time 1, they received $\$ 6.00$ in payment administered directly to participants via Prolific. For the Time 2 and Time 3 follow-up, participants from Time 1 were recontacted via the Prolific website. At both follow-up periods, potential participants were again provided with a brief description of the survey and offered additional payment at each time point. Participants were notified that they had previously completed a similar survey and were asked to complete the follow-up survey. The average completion time for Time 1 was 33 minutes (range: 7 to 80 minutes); 32 minutes (range: 7 to 142 minutes) at Time 2; and 29 minutes (range: 6 to 99 minutes) at Time 3. To ensure the quality of the data, three attention checks were embedded throughout the survey. For all three time points, none of the participants failed more than one of the attention checks.

All contacts with participants were conducted via the Prolific survey research website. Contacts were anonymized and participants were recontacted via a nonidentifying Prolific ID number. All data provided to the research team were anonymous and contained no identifying information. Because of the anonymous, deidentified nature of the data, this study was deemed exempt from oversight by the University of Michigan Institutional Review Board.

\section{Participants}

This was a national survey, with participants located throughout the United States. A total of 555 adults residing in the United States completed the survey. The analytic sample for the current study included participants who indicated they were in an intimate relationship at Time 1, Time 2, and Time $3(N$ $=291$, or $52.4 \%$ of the total sample). As seen in Table 1, the average age was 
37 years. The majority had at least a bachelor's degree (61\%) and identified as White (non-Hispanic; 77\%); 7\% identified as Black; $8 \%$ identified as Hispanic; $8 \%$ identified in another category of race. Average household income in the prior year was between $\$ 50,000$ and $\$ 70,000$.

\section{Dependent Variable Measures}

Relationship conflict during the pandemic. Four questions were posed to assess participants' perceptions of relationship conflict at each time point of data collection. The survey had the prompt: "These questions refer to your romantic relationship." At Time 1, participants were given the prompt, "In the last 2 weeks, since the Coronavirus/COVID-19 global health crisis:" and for Time 2 and Time 3, participants were given the prompt, "In the last 2 weeks, during the Coronavirus/ COVID-19 global health crisis:" For the item assessing disagreements related to COVID-19, participants were asked, "My spouse or partner and I have had disagreements related to the Coronavirus/ Covid-19." For the item assessing perception of having more disagreements than usual, participants were asked, "My spouse or partner and I have had more disagreements than usual." For the item assessing verbal fights, "My spouse or partner and I have had more verbal fights than usual." For the item assessing physical fights, participants were asked, "My spouse or partner and I have had more physical fights than usual." Each item was rated on a scale from 1 (strongly disagree) to 5 (strongly agree).

\section{Independent Variables}

Dyadic coping scale. At Time 1, 10 items from the supportive dyadic coping subscale of the Dyadic Coping Inventory $(\alpha=.85$; Bodenmann, 2008; Ledermann et al., 2010) assessed coping that occurs when one or both partners are stressed. The stem was: "This section is about what your partner did when you were feeling stressed. During the past 2 weeks, since the Coronavirus/COVID-19 global health crisis." Items included: "My partner showed empathy and understanding to me," "My partner expressed they were on my side," "My partner took on things that I normally do to help me out," and "My partner helped me to see stressful situations in a different light," rated from 1 (very rarely) to 5 (very often) and averaged with higher scores indicating stronger dyadic coping.

Increased alcohol use since the pandemic. Lacking a baseline measure of how frequently individuals used alcohol prior to the pandemic, at Time 1 we asked for participants' self-assessment of increased alcohol use since the 
pandemic, "Have you consumed alcohol (including wine, beer, and cocktails) more than usual since the Coronavirus/COVID-19 global health crisis?" $(0=$ no, 1 = yes, 2 = I do not drink alcohol). For our analyses, participants who do not drink alcohol were coded as 0 given they had not increased their alcohol use.

Depression. At Time 1, depression was measured using the eight-item Personal Health Questionnaire (PHQ- 8; Kroenke et al., 2009), which is a valid diagnostic tool that measures severity of depressive disorders in the general population. Participants were asked, "Over the last two weeks, how often have you been bothered by any of the following problems?" Sample items included, "Little interest or pleasure in doing things," "Feeling down, depressed, or hopeless," and "Feeling tired or having little energy." Items were assessed on a 4 -point scale $(0=$ not at all, $1=$ several days, $2=$ more than half the days, and $3=$ nearly every day), resulting in a score range from 0 to 24. A score of 9 or under indicates the participant is not depressed; a score between 10 and 19 is indicative of probable major depression; and a score between 20 and 24 is indicative of probable severe major depression. We created a dichotomous variable to reflect whether the participant met the PHQ -8 criteria for major depression or severe major depression, in which scores of 9 or less were coded " 0 " and scores of 10 or above were coded " 1 " $(0=$ not depressed, $1=$ probable major depression or severe major depression).

Anxiety. At Time 1, anxiety was measured with the Generalized Anxiety Disorder seven-item scale (GAD-7; Spitzer et al., 2006), a valid diagnostic tool to measure anxiety symptoms in the general population. Participants were asked, "Over the last 2 weeks, how often have you been bothered by the following problems?" Sample items include, "Feeling nervous, anxious, or on-edge," "not being able to stop or control worrying," and "trouble relaxing." Items were rated on a 4-point scale $(0=$ not at all, $1=$ several days, 2 $=$ more than half the days, and $3=$ nearly every day), resulting in a score range from 0 to 21 . A score of 4 or less indicates minimal anxiety; a score between 5 and 9 indicates probable mild anxiety; a score between 10 and 14 indicates probable moderate anxiety; and a score between 15 and 21 indicates probable severe anxiety. We created a dichotomous variable to indicate probable moderate or severe anxiety. Scores of 9 or less were coded " 0 " and scores of 10 or above were coded " 1 " $(0=$ minimal or mild anxiety, $1=$ moderate or severe anxiety).

Employment change due to COVID-19. At Time 1, a dichotomous variable indicated whether participants had experienced an employment change due to COVID-19: "Has your employment status changed (e.g., laid off, furloughed) in the last 2 weeks because of the Coronavirus/COVID-19 global health crisis?" $(0=$ no, $1=$ yes $)$. 
Days in lockdown. A Time 1, participants were also asked, "Are you currently engaging in "lockdown" (e.g., sheltering-in-place, only going out for essential activities)?" If yes, "About how many days ago did you go on lockdown?" This variable is continuous.

\section{Control Variables}

Sociodemographic controls. Controls were measured at Time 1. Race was measured using questions from the U.S. Census. Participants indicated whether they were of Hispanic, Latino, or Spanish ethnic origin, and then identified their race. Because $77 \%$ of participants identified as Non-Hispanic White, for ease of interpretation and to avoid drawing conclusions on racial groups whose sample sizes were small, analyses used a dichotomously coded variable $(0=$ White [Non-Hispanic], $1=$ Non-White $)$. Education level was modeled as a series of dummy variables (high school degree or less [comparison], some college, college degree or higher). Age was continuous and measured in years. Sex was dichotomous $(0=$ male, $1=$ female $)$. Total household income in the last year before taxes was also treated as continuous, justified by the large number of categories that were measured $(1=\$ 10-20 k, 2=\$ 20-30$ $k, 3=\$ 30-40 k, 4=\$ 40-50 k, 5=\$ 50-70 k, 6=\$ 70-90 k, 7=\$ 90 k$ or more $)$.

\section{Analysis Plan}

Data cleaning and descriptive analyses were run in Stata version 15.1. Longitudinal analyses were run in Mplus version 8 (Muthén \& Muthén, 1998-2017). To examine the changes in the four dependent variables over time, we conducted linear piecewise growth models in Mplus using Bayesian estimation. Piecewise models tend to produce the statistical power needed to detect differences in slope estimates in small sample sizes, produce accurate slope estimates in the presence of missing data, and permit the examination of change over time (Curran et al., 2010; Fan, 2003). Bayesian estimation was advantageous in that it allowed us to estimate more parameters, and thus a more complex model, with a smaller sample size than is possible using frequentist statistics. Noninformative priors were utilized, meaning that assumptions were not imposed on the parameters estimated in the model. Noninformative priors are generally preferred when prior information about a sample or effects are scarce or unavailable (van de Schoot et al., 2014). Data were scanned for outliers, multicollinearity, and missingness. No outliers or instances of multicollinearity were identified. There were few missing data on our dependent variables across all three time points $(<1 \%)$. On our 
independent variables, there were no missing data on dyadic coping or alcohol use, and approximately $1 \%$ missing data on anxiety and depression. Our control variables also had minimal missing data $(<3 \%)$. To handle missing data, analyses were conducted using full information maximum likelihood estimation (FIML) analyses, which uses all available data and permits consistency of sample size across models.

We tested four models, one for each dependent variable. For the first research question, we ran models with the dependent variables only ("initial model") to test whether these scores changed over time. Next, we introduced Time 1 sociodemographic controls and the independent variables into the model ("full model"), which allowed us to determine which variables influenced the intercept (i.e., the mean scores at Time 1) and the slope (i.e., change in scores over time) of the dependent variables. Model fit was evaluated using the Posterior Predictive $p$ (PPP) value, where values greater than .05 indicate good fit (Elsworth et al., 2016).

\section{Results}

\section{Descriptive Statistics}

As seen in Table 1, at Time 1, one in four study participants met the PHQ-8 criteria for probable major or severe major depression; $32.64 \%$ met the GAD -7 criteria for moderate or severe anxiety. $19.24 \%$ reported an employment change due to COVID-19. More disagreements than usual was the most common form of relationship conflict. More physical fights than usual was the least common form of relationship conflict.

Table I. Descriptive Statistics of Study Variables $(n=291)$.

\begin{tabular}{|c|c|c|c|c|c|c|}
\hline & $M$ & SD & Min & $\operatorname{Max}$ & $n$ & $\%$ \\
\hline $\begin{array}{l}\text { Major or severe } \\
\text { depression, Time I }\end{array}$ & & & & & 75 & 25.95 \\
\hline $\begin{array}{l}\text { Moderate or severe } \\
\text { anxiety, Time I }\end{array}$ & & & & & 94 & 32.64 \\
\hline $\begin{array}{l}\text { Increased alcohol use } \\
\text { since the pandemic, } \\
\text { Time I }\end{array}$ & & & & & 44 & 17.74 \\
\hline $\begin{array}{l}\text { Employment status } \\
\text { changed due to } \\
\text { COVID-19, Time I }\end{array}$ & & & & & 56 & 19.24 \\
\hline $\begin{array}{l}\text { White (Non-Hispanic), } \\
\text { Time I }\end{array}$ & & & & & 222 & 76.82 \\
\hline
\end{tabular}


Table 1. continued

\begin{tabular}{|c|c|c|c|c|c|c|}
\hline & M & SD & Min & Max & $\mathrm{n}$ & $\%$ \\
\hline \multicolumn{7}{|l|}{ Education, Time I } \\
\hline High school or less & & & & & 28 & 9.62 \\
\hline Some college & & & & & 86 & 29.55 \\
\hline College degree or higher & & & & & 177 & 60.82 \\
\hline Dyadic coping, Time I & 3.68 & 0.74 & 1.6 & 5 & & \\
\hline Age, Time I & 36.64 & 10.04 & 19 & 7I & & \\
\hline Income, Time I & 4.99 & 1.88 & I & 7 & & \\
\hline $\begin{array}{l}\text { Days spent in lockdown, } \\
\text { Time I }\end{array}$ & 5.55 & 4.71 & 0 & 25 & & \\
\hline $\begin{array}{l}\text { Disagreements related to } \\
\text { COVID-19, Time I }\end{array}$ & 2.05 & 1.24 & I & 5 & & \\
\hline $\begin{array}{l}\text { Disagreements related to } \\
\text { COVID-19, Time } 2\end{array}$ & 2.07 & 1.23 & I & 5 & & \\
\hline $\begin{array}{l}\text { Disagreements related to } \\
\text { COVID-19, Time } 3\end{array}$ & 2.02 & 1.22 & I & 5 & & \\
\hline $\begin{array}{l}\text { More disagreements than } \\
\text { usual, Time I }\end{array}$ & 1.97 & 1.21 & I & 5 & & \\
\hline $\begin{array}{l}\text { More disagreements than } \\
\text { usual, Time } 2\end{array}$ & 2.19 & 1.25 & I & 5 & & \\
\hline $\begin{array}{l}\text { More disagreements than } \\
\text { usual, Time } 3\end{array}$ & 2.19 & 1.27 & I & 5 & & \\
\hline $\begin{array}{l}\text { More verbal fights than } \\
\text { usual, Time I }\end{array}$ & 1.86 & 1.16 & I & 5 & & \\
\hline $\begin{array}{l}\text { More verbal fights than } \\
\text { usual, Time } 2\end{array}$ & 2.00 & 1.18 & 1 & 5 & & \\
\hline $\begin{array}{l}\text { More verbal fights than } \\
\text { usual, Time } 3\end{array}$ & 2.03 & 1.22 & 1 & 5 & & \\
\hline $\begin{array}{l}\text { More physical fights than } \\
\text { usual, Time I }\end{array}$ & 1.19 & 0.57 & 1 & 5 & & \\
\hline $\begin{array}{l}\text { More physical fights than } \\
\text { usual, Time } 2\end{array}$ & 1.24 & 0.61 & 1 & 5 & & \\
\hline $\begin{array}{l}\text { More physical fights than } \\
\text { usual, Time } 3\end{array}$ & 1.25 & 0.57 & I & 5 & & \\
\hline
\end{tabular}

Note. Income categories: $I=\$ 10-20 k, 2=\$ 20-30 k, 3=\$ 30-40 k, 4=\$ 40-50 k, 5=\$ 50-70 k$, $6=\$ 70-90 k, 7=\$ 90 k$ or more. Comparison category for depression is "not depressed." Comparison category for anxiety is "minimal or mild anxiety." Comparison category for White is non-White. 


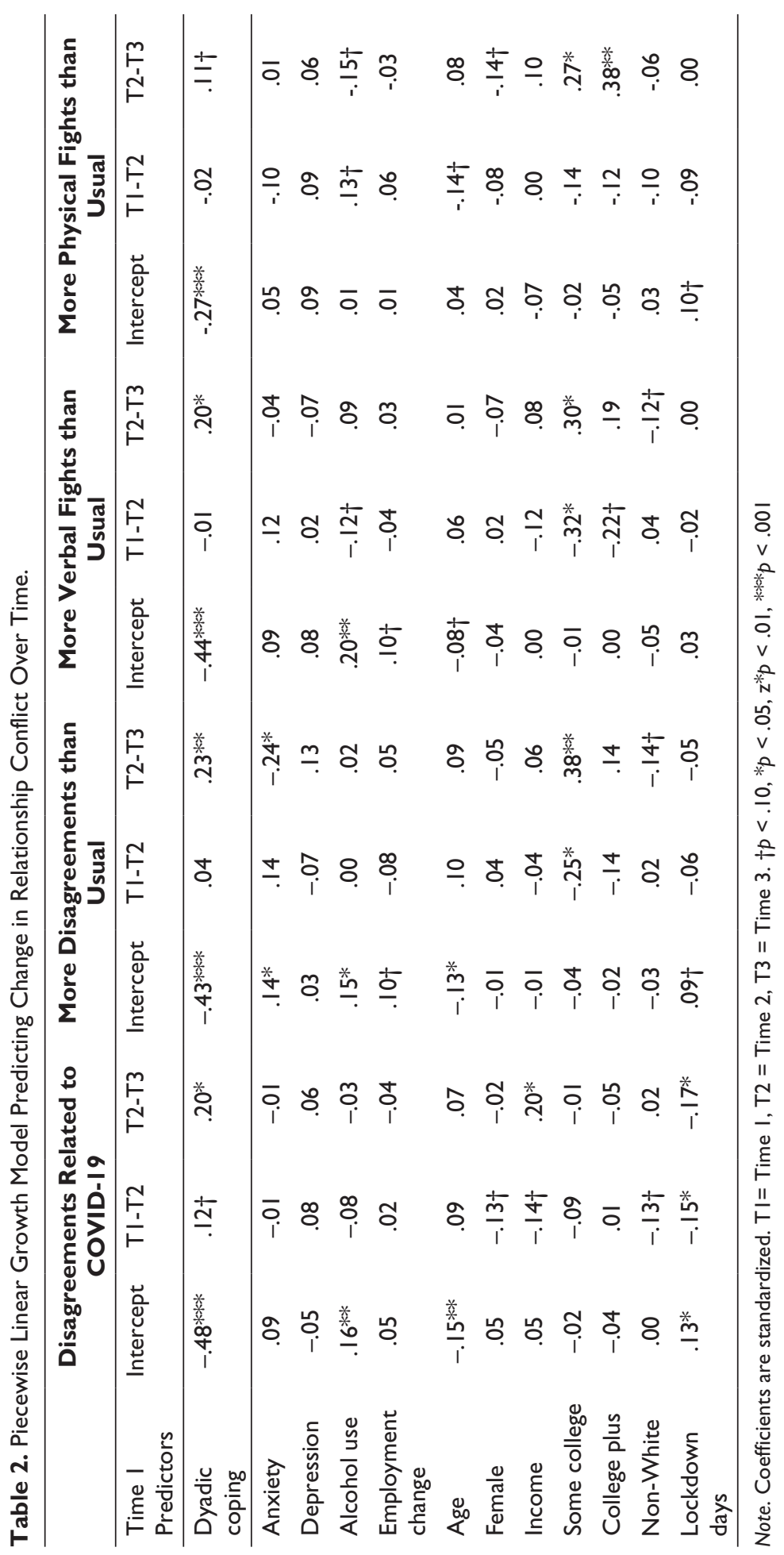




\section{Couples' Disagreements Related to COVID-19}

The initial model analyzing couples' disagreements related to COVID-19 had good fit ( $\mathrm{PPP}=.47)$. There was no statistically significant change in couples' disagreements related to COVID-19 from Time 1 to Time $2(B=0.02, p=$ $.399)$ or from Time 2 to Time $3(B=-.07, p=.217$; see Figure 1$)$. The full model also had good fit $(\mathrm{PPP}=.35)$. Dyadic coping $(B=-.48, p<.001)$ was negatively associated with the intercept, suggesting that those with stronger dyadic coping reported less disagreements related to COVID-19 on average at Time 1. The number of lockdown days $(B=.13, p=.013)$ and increased alcohol use since the pandemic $(B=.16, p=.003)$ were positively associated with the intercept, suggesting that those with longer lockdown and greater alcohol use reported more disagreements at Time 1. Number of lockdown days was negatively associated with the change in couples' disagreements related to COVID-19 from Time 1 to Time $2(B=-.15, p=.037)$; longer lockdown decreased the slope of disagreements related to COVID-19 from Time 1 to Time 2. Although the overall slope of disagreements from Time 2 to Time 3 was nonsignificant, dyadic coping $(B=.20, p=.014)$ was positively associated with the change in couples' disagreements related to

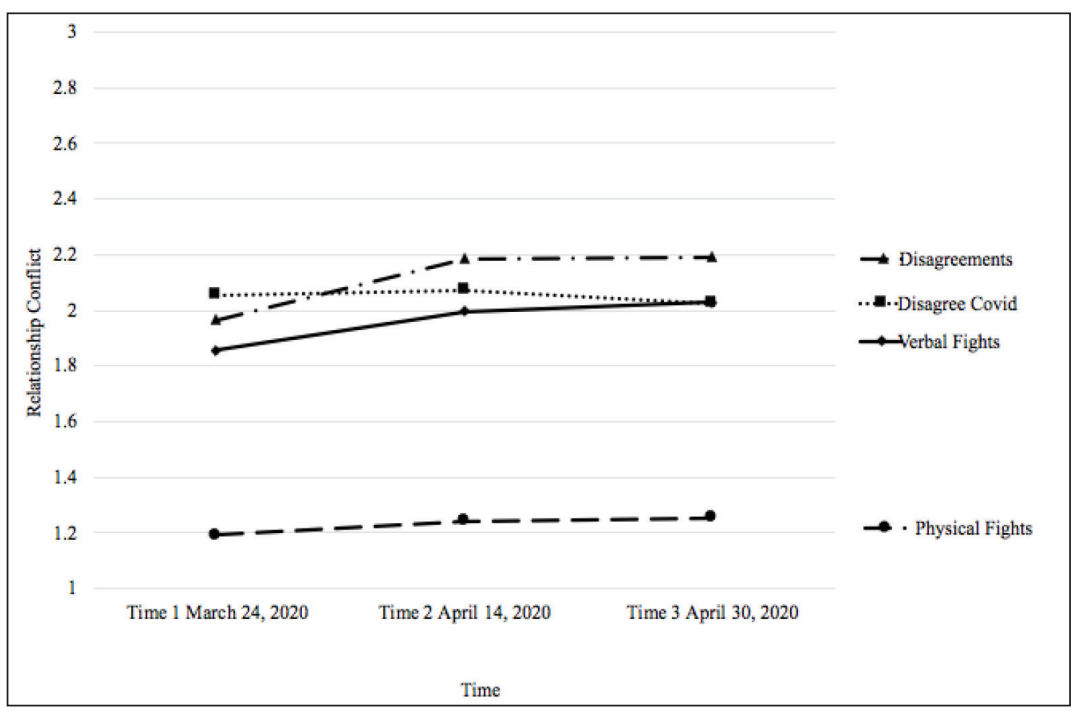

Figure I. Changes in relationship conflict across time $(N=291)$.

Note. The scale for relationship conflict items was from I (strongly disagree) to 5 (strongly agree). The $\mathrm{Y}$-axis presents the average score for all study participants. The $\mathrm{X}$-axis presents the time point of data collection. 
COVID-19 from Time 2 to Time 3, wherein stronger dyadic coping at Time 1 increased the slope of disagreements related to COVID-19 from Time 2 to Time 3. Because those endorsing stronger dyadic coping reported less COVID-19 disagreements at Time 1, these individuals had greater likelihood of increasing disagreements over time.

\section{Couples Having More Disagreements Than Usual}

The initial model examining couples having more disagreements than usual had good fit (PPP $=.47)$. There was a statistically significant increase in couples having more disagreements than usual from Time 1 to Time $2(B=$ $.31, p<.001)$, but not from Time 2 to Time $3(B=.01, p=.455$; see Figure $1)$. The full model also had good fit ( $\mathrm{PPP}=.34)$. Moderate or severe anxiety $(B=.14, p=.044)$ and increased alcohol use since the pandemic $(B=.15, p$ $=.010$ ) were positively associated with the intercept; in other words, individuals with high anxiety and increased alcohol use reported more disagreements at Time 1 . Having moderate or severe anxiety at Time 1 was inversely associated with change in disagreement scores from Time 2 to Time $3(B=$ $-.24, p=.017)$; in other words, high anxiety levels at Time 1 was associated with a decrease in the slope of disagreements from Time 2 to Time 3. Dyadic coping $(B=-.43, p<.001)$ was negatively associated with the intercept suggesting that individuals with higher dyadic coping scores reported fewer disagreements at Time 1 . Dyadic coping at Time $1(B=.23, p=.005)$ was positively associated with change in disagreement scores from Time 2 to Time 3. Higher Time 1 dyadic coping scores were associated with increased slope of disagreements from Time 2 to Time 3 . As individuals with high anxiety reported more disagreements at Time 1 , and individuals with stronger dyadic coping reported less disagreements at Time 1, these individuals had greater potential to experience decreases or increases in disagreements over time, respectively.

\section{More Verbal Fights Than Usual}

The initial model analyzing couples having more verbal fights than usual had good fit $(\mathrm{PPP}=.48)$. There was a statistically significant difference in verbal fighting from Time 1 to Time $2(B=.22, p=.009)$, but not from Time 2 to Time $3(B=.04, p=.316$; see Figure 1). The full model also had good fit (PPP $=.34)$. Increased alcohol use was positively associated with the intercept $(B=$ $.20, p=.001)$ wherein those who increased alcohol use reported more verbal fights at Time 1. Dyadic coping was negatively associated with the intercept $(B=-.44, p<.001)$. Individuals with stronger dyadic coping reported fewer 
verbal fights at Time 1, but dyadic coping was positively associated with change (increase) in verbal fighting from Time 2 to Time $3(B=.20, p=.013)$, although the overall slope between Time 2 to Time 3 was nonsignificant.

\section{More Physical Fights Than Usual}

The initial model analyzing having more physical fights than usual had good fit $(\mathrm{PPP}=.47)$. There was not a statistically significant difference in physical fights from Time 1 to Time $2(B=.12, p=.084)$ or from Time 2 to Time 3 ( $B$ $=.03, p=.352$; see Figure 1). The full model also had good fit $(\mathrm{PPP}=.34)$. Dyadic coping was negatively associated with the intercept $(B=-.27, p<$ .001 ), meaning that individuals with stronger dyadic coping reported less physical fights at Time 1 . None of our primary independent variables of interest were associated with changes in physical fighting scores from Time 1 to Time 2 or from Time 2 to Time 3 .

\section{Discussion}

Natural disasters and economic recessions are associated with increases in rates of family violence (Buttell \& Ferreira, 2020; Harville et al., 2011; Hawkins, 2009; Schneider et al., 2016; Serrata \& Alvarado, 2019). Based on prior research, the risk factors of interest included recent unemployment, mental health problems (depression and anxiety), and alcohol use. Given that quarantine may potentially exacerbate conflict (Mazza et al., 2020), we also examined the number of days in lockdown. We considered dyadic coping as a resilience factor. Analysis of longitudinal survey data suggested that among this well-educated sample, levels of relationship conflict were fairly low. Participants' reports of having more disagreements than usual and having more verbal fights than usual increased significantly over time, whereas reports of disagreements related to COVID-19 and physical fights did not increase over time.

This study provides some support for the notion that coping skills can help to mitigate stressful conditions during the pandemic (Chen \& Bonanno, 2020). As hypothesized, and consistent with prior studies (Bodenmann, 2000; Falconier \& Kuhn, 2019), perceived dyadic coping was a protective factor for all relationship conflict scores at Time 1 (i.e., toward the beginning of the pandemic), even in controlling for high levels of job loss due to COVID-19 (nearly one in five study participants) and mental health concerns, with $25.95 \%$ of participants reporting symptoms that met the PHQ-8 criteria for probable major or severe major depression and $32.64 \%$ of participants reporting symptoms that met the GAD -7 criteria for moderate or severe anxiety. Notably, 
although rates of anxiety and depression were high among study participants, they were consistent with other studies showing a dramatic increase in the levels of anxiety and depression during the pandemic (Twenge \& Joiner, 2021).

However, contrary to our hypotheses, stronger dyadic coping did not seem to buffer individuals against short-term increases in conflict. Although individuals who reported better dyadic coping started the pandemic with lower levels of disagreements and fighting, stronger dyadic coping at Time 1 was associated with an increase in the slope of disagreements and verbal fighting from Time 2 to Time 3. Because individuals with stronger dyadic coping reported less conflict at Time 1, these individuals may have had greater potential to experience increases in disagreements over time.

Alternatively, adults may have overestimated their dyadic coping early in the pandemic whereby those initial judgments about dyadic coping may have shifted, no longer reflecting dyadic coping processes later in the pandemic. The conditions from the pandemic were unpredictable and worsening during the time these surveys were administered, potentially exacerbating stressful life conditions for study participants. It may be that the study time frame, from March to April 2020, was too short to detect the long-term benefits of dyadic coping.

\section{Implications for Policy, Practice, and Intervention}

This "pandemic within a pandemic" (Evans et al., 2020) of increasing IPV during COVID-19 may be indicative of increasing rates of family violence overall, including child abuse (Lee, Ward, Lee, et al., 2021; Rodriguez et al., 2020), elder abuse (Makaroun et al., 2020), and sibling abuse (Perkins et al., 2021). Vulnerable populations such as immigrants, the LGBTQ population, women of color, and those with disabilities are likely to be disproportionality impacted by increases in family conflict (Rai et al., 2020; Ramaswamy et al., 2020). Studies raise concerns about increases in victimization (Jetelina et al., 2020), whether police are responding adequately to increased IPV calls (Bullinger et al., 2020), and increasing severity of IPV injuries (Gosangi et al., 2020) during COVID-19. Thus, one implication is the need to ensure effective intervention and response during the pandemic.

From a policy and practice standpoint, there are a number of potential avenues to address family violence. It is likely that the social service network is going to be strained by the need to provide services to larger numbers of victims and survivors. Thus, one implication is for enhanced funding of the Violence Against Women Act to support services such as emergency shelter and transitional housing (Ramaswamy et al., 2020). Another initiative that would benefit IPV victims and survivors is employer-paid safe leave, which 
would allow victims and survivors time off from work in crisis situations (Ramaswamy et al., 2020).

Although there may be limitations to the use of telehealth for victims of IPV, given safety concerns with abusers potentially present during calls, the use of telehealth for some intervention services and mental health services is promising and may be a practical solution given the social distancing strictures related to COVID-19 (Moreno et al., 2020; Ramaswamy et al., 2020). Social media is another emerging strategy for awareness campaigns. One study examined Twitter hashtags as a mechanism to distribute evidence-based information about sexual abuse (Wekerle et al., 2018). Another study utilized Reddit to examine the concerns of foster families during COVID-19 (Lee, Chang et al.,2021). Smartphone apps such as the myPlan app are designed for safety planning for partner violence. Social media may be an effective way to disseminate educational information and media campaigns. Social media may also be a way to anonymously raise awareness about resources and programs, and even help women to engage in safety planning.

\section{Study Limitations}

Because the study utilized a convenience sample, the results of this study may not be generalizable to the broader U.S. population. Participants were not highly diverse. The sample in the current study was $77 \%$ White (NonHispanic), which is comparable to the U.S. Census report for July 2019, of $76.3 \%$ of the population as a whole (U.S. Census Bureau, 2020). However, study participants had higher levels of education, with $61 \%$ having at least a bachelor's degree, compared to $31.5 \%$ of the U.S. population reporting a bachelor's degree or higher. Thus, this study likely does not capture the experiences of individuals who are the most adversely impacted by COVID-19, such as individuals with lower socioeconomic status or lower educational attainment. Another notable limitation of the lack of generalizability of the study results is that it is entirely United States based. The results of this study do not speak to the experiences of individuals in other contexts, such as the international context, even though the effects of COVID-19 are being felt on a global scale. It is important to replicate the study with a more diverse population, particularly individuals who may have experienced higher levels of economic strain. Relatedly, researchers should also consider the experiences of couples in in low- and middle- income countries throughout the world, who may face vastly different economic, social, and relationship challenges during the pandemic.

The small sample size may have contributed to insufficient statistical power to estimate small effect sizes. The data in this study examines the first 
two months of the stay-at-home orders and thus is unable to address long-term trends in relationship conflict. Moreover, the current analyses do not incorporate changes in the primary independent variables of interest (mental health problems, alcohol use, lockdown days, employment change, dyadic coping) which could have also changed across these early weeks of the pandemic. Furthermore, our analyses were based on perceptions of relationship conflict of one partner and are subject to self-presentation biases that are inherent in self-report data. We did not capture the transactional nature of conflict and coping during the pandemic. A more robust study would include both partners' perceptions of conflict to more fully capture dyadic coping processes.

\section{Conclusion}

The current study provides a short-term perspective on relationship conflict during the first weeks of the COVID-19 pandemic. Although study participants reported high levels of disruption, dyadic coping was associated with lower levels of initial relationship conflict. Even so, dyadic coping did not buffer individuals from increases in relationship conflict. With the conditions of the pandemic persisting into the foreseeable future, professionals may see a rise in individuals seeking consultation for relationship conflict. Telehealth options may help to address mental health services (Moreno et al., 2020), but are not an adequate solution for many IPV victims. Future research is needed to examine how couples adapt to the pandemic and whether the ongoing strain of the pandemic contributes to a worsening of conflict over the long-term.

\section{Acknowledgments}

The authors would like to thank Deborah Schild for conducting expedited Institutional Review Board processes for this study, and Joyce Y. Lee and Olivia D. Chang for assistance with this research.

\section{Declaration of Conflicting Interests}

The author(s) declared no potential conflicts of interest with respect to the research, authorship and/or publication of this article.

\section{Funding}

The author(s) disclosed receipt of the following financial support for the research, authorship and/or publication of this article: This study was funded in part by the University of Michigan School of Social Work. 


\section{ORCID iDs}

Shawna J. Lee iD https://orcid.org/0000-0003-0562-2856

Christina M. Rodriguez iD https://orcid.org/0000-0002-5090-0707

\section{References}

Bodenmann, G. (1997). Dyadic coping: A systemic-transactional view of stress and coping among couples: Theory and empirical findings. European Review of Applied Psychology, 47(2), 137-140.

Bodenmann, G. (2000). Stress und coping bei paaren [Stress and coping in couples]. Hogrefe.

Bodenmann, G. (2008). Dyadisches coping inventar: Test manual [Dyadic coping inventory: Test manual]. Huber.

Bodenmann, G., \& Cina, A. (2006). Stress and coping among stable-satisfied, stable-distressed and separated/divorced swiss couples: A 5-year prospective longitudinal study. Journal of Divorce \& Remarriage, 44(1-2), 71-89. https://doi. org/10.1300/J087v44n01_04

Bodenmann, G., Meuwly, N., Bradbury, T. N., Gmelch, S., \& Ledermann, T. (2010). Stress, anger, and verbal aggression in intimate relationships: Moderating effects of individual and dyadic coping. Journal of Social and Personal Relationships, 27(3), 408-424. https://doi.org/10.1177/0265407510361616

Bullinger, L. R., Carr, J. B., \& Packham, A. (2020). COVID-19 and crime: The effects of stay-at-home orders on domestic violence. NBER Working Papers 27667, National Bureau of Economic Research, Inc.

Buttell, F., \& Ferreira, R. J. (2020). The hidden disaster of COVID-19: Intimate partner violence. Psychological Trauma: Theory, Research, Practice, and Policy, 12, S197-S198. https://doi.org/https://dx.doi.org/10.1037/tra0000646

Cafferky, B. M., Mendez, M., Anderson, J. R., \& Stith, S. M. (2018). Substance use and intimate partner violence: A meta-analytic review. Psychology of Violence, $8,110-131$.

Capaldi, D. M., Knoble, N. B., Shortt, J. W., \& Kim, H. K. (2012). A systematic review of risk factors for intimate partner violence. Partner Abuse, 3(2), 231-280.

Charles, D., Whitaker, D. J., Le, B., Swahn, M., \& DiClemente, R. J. (2011). Differences between perpetrators of bidirectional and unidirectional physical intimate partner violence. Partner Abuse, 2(3), 344-364.

Chen, S., \& Bonanno, G. A. (2020). Psychological adjustment during the global outbreak of COVID-19: A resilience perspective. Psychological Trauma: Theory, Research, Practice, and Policy, 12, S51-S54. https://doi.org/https://dx.doi. org/10.1037/tra0000685

Colbert, S., Wilkinson, C., Thornton, L., \& Richmond, R. (2020). COVID-19 and alcohol in Australia: Industry changes and public health impacts. Drug and Alcohol Review, 39, 435-440. https://doi.org/10.1111/dar.13092 
Conger, R. D., Conger, K. J., \& Martin, M. J. (2010). Socioeconomic status, family processes, and individual development. Journal of Marriage and Family, 72(3), 685-704. https://doi.org/10.1111/j.1741-3737.2010.00725.x

Cunradi, C. B., Todd, M., Duke, M., \& Ames, G. (2009). Problem drinking, unemployment, and intimate partner violence among a sample of construction industry workers and their partners. Journal of Family Violence, 24, 63-74. https://doi. org/10.1007/s10896-008-9209-0

Curran, P. J., Obeidat, K., \& Losardo, D. (2010). Twelve frequently asked questions about growth curve modeling. Journal of Cognition and Development, 11(2), 121-136. https://doi.org/10.1080/15248371003699969

Elsworth, G. R., Beauchamp, A., \& Osborne, R. H. (2016). Measuring health literacy in community agencies: A Bayesian study of the factor structure and measurement invariance of the health literacy questionnaire (HLQ). BMC Health Services Research, 16, Article 508. https://doi.org/10.1186/s12913-016-1754-2

Evans, M. L., Lindauer, M., \& Farrell, M. E. (2020). A pandemic within a pandemic - intimate partner violence during covid-19. New England Journal of Medicine, 383, 2302-2304. https://doi.org/10.1056/NEJMp2024046

Falconier, M. K., \& Kuhn, R. (2019). Dyadic coping in couples: A conceptual integration and a review of the empirical literature. Frontiers in Psychology, 10, 571. https://doi.org/10.3389/fpsyg.2019.00571

Fan, F. (2003). Power of latent growth modeling for detecting group differences and growth trajectory parameters. Structural Equation Modeling, 10, 380-400. https://doi.org/10.1207/S15328007SEM1003_3

Foran, H. M., \& O'Leary, K. D. (2008). Alcohol and intimate partner violence: A meta-analytic review. Clinical Psychology Review, 28(7), 1222-1234. https:// doi.org/10.1016/j.cpr.2008.05.001

Forgey, M. A., \& Badger, L. (2010). Patterns of intimate partner violence and associated risk factors among married enlisted female soldiers. Violence and Victims, 25, 45-61. https://doi.org/10.1891/0886-6708.25.1.45

Gosangi, B., Park, H., Thomas, R., Gujrathi, R., Bay, C. P., Raja, A. S., Seltzer, S. E., Balcom, M. C., McDonald, M. L., Orgill, D. P., Harris, M. B., Rexrode, K., \& Khurana, D. (2020). Exacerbation of physical intimate partner violence during COVID-19 lockdown. Radiology, 298(1), E38-E45. https://doi.org/10.1148/ radiol.2020202866

Graham, K., Bernards, S., Wilsnack, S. C., \& Gmel, G. (2011). Alcohol may not cause partner violence but it seems to make it worse: A cross-national comparison of the relationship between alcohol and severity of partner violence. Journal of Interpersonal Violence, 26(8), 1503-1523. https://doi. org/10.1177/0886260510370596

Harville, E. W., Taylor, C. A., Tesfai, H., Xiong, X., \& Buekens, P.(2011). Experience of hurricane Katrina and reported intimate partner violence. Journal of Interpersonal Violence, 26(4), 833-845. https://doi.org/10.1177/0886260510365861 
Hawkins, R. L. (2009). Same at it ever was, only worse: Negative life events and poverty among New Orleans Katrina survivors. Families in Society, 90(4), 375-381. https://journals.sagepub.com/doi/10.1606/1044-3894.3922

Jetelina, K. K., Knell, G., \& Molsberry, R. J. (2020). Changes in intimate partner violence during the early stages of the COVID-19 pandemic in the USA. Injury Prevention, 27(1), 93-97. https://doi.org/10.1136/injuryprev-2020-043831

Kroenke, K., Strine, T. W., Spitzer, R. L., Williams, J. B. W., Berry, J. T., \& Mokdad, A. H. (2009). The PHQ-8 as a measure of current depression in the general population. Journal of Affective Disorders, 114(1-3), 163-173. https://doi. org/10.1016/j.jad.2008.06.026

Ledermann, T., Bodenmann, G., Gagliardi, S., Charvoz, L., Verardi, S., Rossier, J., Bertni, A., \& Lafrate, R. (2010). Psychometrics of the dyadic coping inventory in three language groups. Swiss Journal of Psychology, 69, 201-212. https://doi. org/10.1024/1421-0185/a000024

Lee, J. Y., Chang, O. D., \& Ammari, T. (2021). Using social media reddit data to examine Foster families' concerns and needs during COVID-19. Child Abuse and Neglect.

Lee, S. J., Ward, K. P., Chang, O. D., \& Downing, K. D. (2021). Parenting activities and the transition to home-based education during the COVID-19 pandemic. Children and Youth Services Review, 122, 105585. https://doi.org/10.1016/j. childyouth.2020.105585

Lee, S. J., Ward, K. P., Lee, J. Y., \& Rodriguez, C. M. (2021). Parental social isolation and child maltreatment risk during the COVID-19 pandemic. Journal of Family Violence. Advance online publication. https://doi.org/10.1007/s10896020-00244-3

Levesque, C., Lafontaine, M. F., Caron, A., Flesch, J. L., \& Bjornson, S. (2014). Dyadic empathy, dyadic coping, and relationship satisfaction: A dyadic model. Europe's Journal of Psychology, 10(1), 118-134. https://doi.org/10.5964/ejop. v10i1.697

Makaroun, L. K., Bachrach, R. L., \& Rosland, A. M. (2020). Elder abuse in the time of COVID-19-Increased risks for older adults and their caregivers. The American Journal of Geriatric Psychiatry, 28(8), 876-880. https://doi.org/10.1016/j. jagp.2020.05.017

Mazza, M., Marano, G., Lai, C., Janiri, L., \& Sani, G. (2020). Danger in danger: Interpersonal violence during COVID-19 quarantine. Psychiatry Research, 289, 113046. https://doi.org/10.1016/j.psychres.2020.113046

Merz, C. A., Meuwly, N., Randall, A. K., \& Bodenmann, G. (2014). Engaging in dyadic coping: Buffering the impact of everyday stress on prospective relationship satisfaction. Family Science, 5(1), 30-37. https://doi-org.proxy.lib.umich. edu/10.1080/19424620.2014.927385

Moreno, C., Wykes, T., Galderisi, S., Nordentoft, M., Crossley, N., Jones, N., \& Chen, E. Y. (2020). How mental health care should change as a consequence of the COVID-19 pandemic. The Lancet Psychiatry, 7(9), 813-824. https://doi. org/10.1016/S2215-0366(20)30307-2 
Muthen, L. K., \& Muthen, B. O. (1998-2017). Mplus user's guide (8th ed). Muthen \& Muthen.

Neppl, T. K., Senia, J. M., \& Donnellan, M. B. (2016). Effects of economic hardship: Testing the family stress model over time. Journal of Family Psychology, 30(1), 12-21. https://doi.org/10.1037/fam0000168

Palan, S., \& Schitter, C. (2018). Prolific.Ac-A subject pool for online experiments. Journal of Behavioral and Experimental Finance, 17 22-27. https://doi. org/10.1016/j.jbef.2017.12.004

Perkins, N. H., Rai, A., \& Grossman, S. F. (2021). Physical and emotional sibling violence in the time of COVID-19. Journal of Family Violence. Advance online publication. https://doi.org/10.1007/s10896-021-00249-6

Rai, A., Perkins, N., \& Grossman, S. (2020). The effects of COVID-19 on domestic violence and immigrant families. Greenwich Social Work Review, 2(1), 84-96. https://doi.org/10.21100/gswr.v1i2.1161

Ramaswamy, A., Ranji, U., \& Salganicoff, A. (2020). Finding policy responses to rising intimate partner violence during the coronavirus outbreak. Kaiser Family Foundation. https://www.kff.org/coronavirus-covid-19/issue-brief/finding-policy-responses-to-rising-intimate-partner-violence-during-the-coronavirus-outbreak/

Rodriguez, C. M., Lee, S. J., Ward, K. P., \& Pu, D. F. (2020). The perfect storm: Hidden risk of child maltreatment during the COVID-19 pandemic. Child Maltreatment. Advance online publication. https://doi.org/10.1177/1077559520982066

Schneider, D., Harknett, K., \& McLanahan, S. (2016). Intimate partner violence in the great recession. Demography, 53, 471-505. https://doi.org/10.1007/s13524016-0462-1

Serrata, J. V., \& Alvarado, M. G. H. (2019). Understanding the impact of Hurricane Harvey on family violence survivors in Texas and those who serve them. Texas Council on Family Violence. https:/tcfv.org/wp-content/uploads/2019/08/ Hurricane-Harvey-Report-FINAL-and-APPROVED-as-of-060619.pdf

Smith, S. G., Chen, J., Basile, K. C., Gilbert, L. K., Merrick, M. T., Patel, N., Walling, M., \& Jain, A. (2017). The national intimate partner and sexual violence survey (NISVS): 2010-2012 state report. Centers for Disease Control and Prevention. https://www.cdc.gov/violenceprevention/pdf/NISVS-StateReportBook.pdf

Smith, S. G., Zhang, X., Basile, K. C., Merrick, M. T., Wang, J., Kresnow, M. J., \& Chen, J. (2018). The national intimate partner and sexual violence survey: 2015 data brief-updated release. Centers for Disease Control and Prevention. https:// www.cdc.gov/violenceprevention/pdf/2015data-brief508.pdf

Spencer, C., Mallory, A. B., Cafferky, B. M., Kimmes, J. G., Beck, A. R., \& Stith, S. M. (2019). Mental health factors and intimate partner violence perpetration and victimization: A meta-analysis. Psychology of Violence, 9(1), 1-17. https://doi. org/10.1037/vio0000156

Spitzer, R. L., Kroenke, K., Williams, J. B. W., \& Lowe, B. (2006). A brief measure for measuring generalized anxiety disorder. Archives of Internal Medicine, 166(10), 1092-1097. https://doi.org/10.1001/archinte.166.10.1092 
Stith, S. M., Smih, D. B., Penn, C. E., Ward, D. B., \& Tritt, D. (2004). Intimate partner physical abuse perpetration and victimization risk factors: A meta-analytic review. Aggression and Violent Behavior, 10(1), 65-98. https://doi.org/10.1016/j. avb.2003.09.001

The White House, Office of the Press Secretary. (2020, March 16). The President's coronavirus guidelines for America: 15 days to slow the spread. https://www. justice.gov/doj/page/file/1258511/download

Twenge, J. M., \& Joiner, T. E. (2021). U.S. Census Bureau-assessed prevalence of anxiety and depressive symptoms in 2019 and during the 2020 COVID-19 pandemic. Depression \& Anxiety, 37(10), 954-956. https://doi.org/10.1002/da.23077

U.S. Bureau of Labor Statistics. (2020, May 8). Employment situation summary [News release]. https://www.bls.gov/news.release/empsit.nr0.htm

U.S. Census Bureau (2020, July). Population estimates. https://www.census.gov/ quickfacts/fact/table/US/PST045219

van de Schoot, R., Kaplan, D., Denissen, J., Asendorpf, J. B., Neyer, F. J., \& van Aken, M. A. G. (2014). A gentle introduction to Bayesian analysis: Applications to developmental research. Child Development, 85(3), 842-860. https://doi. org/10.1111/cdev.12169

Wekerle, C., Vakili, N., Stewart, S. H., \& Black, T. (2018). The utility of twitter as a tool for increasing reach of research on sexual violence. Child Abuse \& Neglect, $85,220-228$.

World Health Organization. (2020, April 27). WHO timeline - COVID-19. Author. https://www.who.int/news-room/detail/27-04-2020-who-timeline-covid-19

\section{Author Biographies}

Shawna J. Lee, PhD, is Associate Professor at the School of Social Work, University of Michigan, Ann Arbor. She is the director of the Parenting in Context Research Lab. Her research interests include IPV, child maltreatment, and child well-being.

Kaitlin P. Ward, MSW, is a Joint PhD student in Social Work and Developmental Psychology at the University of Michigan, Ann Arbor. Her research focuses on parenting and child well-being.

Christina M. Rodriguez, PhD, is a Professor of Clinical Psychology at the University of Alabama at Birmingham. She conducts research on family violence, including the assessment and prediction of physical child abuse risk and IPV. 